\section{A report from the World Congress on Controversies in Neurology}

Kurt A Jellinger*
The 6th World Congress on Controversies in Neurology, Vienna, Austria, 8-11 March 2012

The 6th World Congress on Controversies in Neurology, held in Vienna, Austria, in March 2012, provided a forum for clinicians and neuroscientists to discuss the advantages and disadvantages of a large spectrum of neurological and neuropsychiatric disorders. With 35 sessions, 65 debates (in four parallel sessions), two satellite symposia and poster sessions, the meeting highlighted the current diagnostic and management criteria across many areas of clinical neurology, with a wide range from multiple sclerosis, neuroimmunology, dementia, brain injury, neurooncology, neurogenesis, stroke, epilepsy, neurorehabilitation, movement disorders and neuropsychiatry. With a multidisciplinary approach, the conference aimed to clarify a myriad of controversial aspects in the pathogenesis, diagnosis, treatment and prevention of major neurological and neuropsychiatric diseases, in order to gain new insights into essential problems and to develop new treatment options for these disorders.
The 6th World Congress on Controversies in Neurology (chairmen: Amos D Korczyn [Tel Aviv, Israel] and Michael Brainin [Krems, Austria]), with over 600 participants from 52 countries, provided 4 days of intensive talks and discussions on a broad range of problems in clinical neurology and neuroscience. As there were so many topics discussed, this report can only highlight a few essential areas that were brought up during the 4 days.

Before the onset of the congress and after a key-note talk from Jeffrey M Schwartz (CA, USA) on the brain and the relationships between religion, cultural backgrounds and attention/self-control, a satellite symposium of the Society for the Study of Neuroprotection and Neuroplasticity, was dedicated to long-term outcomes and treatment options after traumatic brain injuries. The congress opened with talks about the neuroanatomical basis of the psychoanalytical theory (Israel Steiner, [Tel Aviv, Israel]) and the current validity of Freud's neuroscience discoveries (Michael Brainin). A stimulating discussion concerned the questions on whether cerebrospinal fluid analysis and MRI are still necessary for the diagnosis and therapeutic decisions in multiple sclerosis (MS), the option to treat clinical isolated symptoms and whether it is possible to assess the benefits and risks of new disease-modifying therapies. Special sections discussed the relationships between neuromyelitis optica and MS, which are currently considered distinct entities within the broad spectrum of autoimmune disorders (Brian G Weinshenker, MN, USA), new players in MS therapy
*Institute of Clinical Neurobiology, Kenyongasse 18, A-1070 Vienna, Austria; Tel.: +43 1526 6534; Fax: +43 1526 6534; kurt.jellinger@univie.ac.at
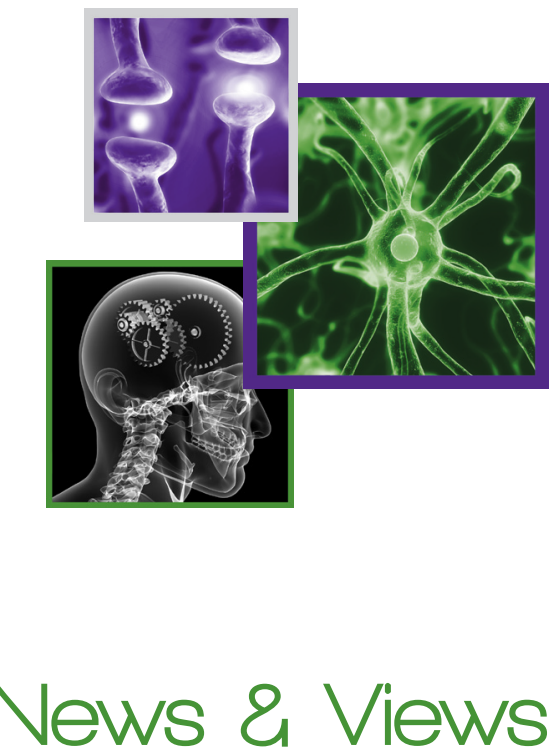

News

Journal Watch

Ask the Experts

Conference Scene 
(e.g., alemtuzumab), the safety of diseasemodifying MS therapies in pregnancy and the possibilities of stimulating repair or control damage by current or future MS therapies. One major section - dementia - included vivid discussions on the benefits of music therapy and possible modification of synaptic loss and cognitive impairment by nutrient mixtures. The value of one valid biomarker for Alzheimer's disease (AD) was critically discussed, but, according to current experience, a combination of biomarkers is needed (Panteleimon Giannokopoulos [Lausanne, Switzerland] vs Peter Riederer [Würzburg, Germany]), as were the causal relationships between inflammation and dementia; inflammation as a factor in a vicious circle was documented by multiple studies, whereas anti-inflammatory drugs failed in modifying dementia. Important subjects of discussion were the question of whether early diagnosis of neurodegenerative disorders by means of preclinical biomarkers may be beneficial in view of successful treatment options being unavailable and whether it is possible to detect incipient or early stages of AD dementia by currently available clinical and imaging methods. The clincial helpfulness and the limits of amyloid imaging (confirmer Roger Bullock, London, UK) and the current knowledge on the role of amyloid aggregates in AD pathogenesis and amyloid as a false target in AD therapy (Ezio Giacobini [Geneva, Switherland] vs Johannes Thome [Rostock, Germany]) were subjects of eager discussion. For neurorehabilitation, the impact of monomodal and multimodal strategies on functional recovery, and the use of antidepressants in poststroke rehabilitation, the clinical value of biomarkers as indicators of cerebral damage after traumatic brain injury and the effects of surgical procedures and pharmaceutical interventions in supporting brain protection and recovery after traumatic brain injury were debated. In the section on neuropsychiatry, there was a discussion as to whether aggression and antisocial behavior could be explained by neurobiological factors such as hormones, neurotransmitters and neuromodulators, (positive answer
Matthäus Willeit, neurologist [Innsbruck, Austria] vs negative answer psychologist Alfred Pritz [Vienna, Austria]), and whether functional symptoms have neurobiological substrates or somatization is a coping mechanism learned from early parent-child interactions. Further points of debate were the effectiveness of transcranial magnetic stimulation in neuropsychiatric disorders, and the presence of cognitive dysfunction in early Parkinson's disease (PD), a fact repeatedly documented by modern methods. The effects of antidepressant drugs on hippocampal neurogenesis as crucial components for future drug development, as well as for understanding functional plasticity of the brain were either confirmed or questioned. In the section of neuro-oncology (chairs: Wolfgang Grisold [Vienna, Austria] and Peter J Stambrook [OH, USA]), intrathecal versus systemic treatment of leptomeningeal carcinomatosis, the use of steroids (not universally approved), the treatment of glioma in the elderly, the problems of development of new therapeutic agents, and the value of NextGen sequencing in the treatment of glioblastoma were matters of discussion. Other subjects were the role of Chlamydophila infections in neurological and psychiatric diseases, the value of steroids in herpes simplex encephalitis, and in bacterial meningitis (not universally accepted).

A field of extensive discussion was stroke - prevention and treatment trials for poststroke complications (e.g., fatigue, aphasia and spasticity), acute-stroke management (e.g., acute reduction of blood pressure and tissue plasminogen activator prior to thrombectomy, value of augmentation of blood flow and of hypothermia), and whether migraine is primarily a vascular disorder (no: Jens Olesen [Copenhagen, Denmark]), the value of Botox ${ }^{\circledR}$ and antidepressants in migraine, the usefulness of the International Classification of Headache Disorders 2nd Edition diagnostic criteria (yes: Jens Olesen), migraine versus tension-type headache, the usefulness of placebo and calcitonin gene-related peptide receptor blockage in migraine. The section of movement disorders included debates on whether motor symptoms are the major component in $\mathrm{PD}$ (the correct answer may be a major component besides nonmotor signs), whether disease progression can be stopped (the consensus was that levodopa [L-dopa] has modified life expectancy of PD patients), whether dopamine agonists are fundamental in PD treatment (less active but more side effects) and new nondopaminergic treatment is useful (e.g., effective in PD depression). Dyskinesias as an inevitable side effect of L-dopa and methods to improve of L-dopa delivery were answered controversially. Still, unsolved problems are whether PD and multiple system atrophy (MSA) are distinct clinical entities (Georg Ransmayer [Linz, Austria] vs Gregor K Wenning [Innsbruck, Austria]; comment Kurt A Jellinger [Vienna, Austria]: two phenotypes in an overlapping continuum of synucleinopathies) and whether MSA-P and MSA-C are isolated disorders (yes: John Duda [PA, USA]).

A vividly discussed subject concerned various topics of epilepsy, such as the usefulness of new classifications and new neuroimaging techniques in the routine diagnosis, prevention and course-modifying effects, the value of antiepileptic drug combinations instead of surgery, treatment of status epilepticus, valproate treatment during pregnancy and the value of antiepileptic drug serum concentrations in treatment (yes: Gerhard Luef [Innsbruck, Austria]; no: Christoph Baumgartner [Vienna, Austria]).

Hot topics were the mindfulness and clinical relevance of human neuroplasticity, based on many experimental and neurophysiological studies showing positive effects of this new paradigm (moderator: Jeffrey M Schwartz), and the interdisciplinary debate on the possibility and effects of prevention of AD (discussion by experts on biomarkers, epidemiology, drug development and socioeconomic consequences). A special field of discussion were neuroimmunological diseases (with diverse statements for intravenous immunoglobulin vs plasmapheresis and immunodepressants (Marinos Dalakas [London, UK] vs Abhijit Chaudhuri [Romford, UK]) and the role of immune processes in neurodegeneration. 
Debates (yes and no statements plus comments) in all sections were of a high standard; the controversial statements were usually well documented, but several questions can not be definitively answered at present, since there is frequent overlap between the facts or definite solutions are not yet possible. This international and interdisciplinary meeting provided the podium for vivid and fruitful discussions on many hitherto unresolved problems in basic and clinical neurology. The next Congress will be held in Istanbul, Turkey, in April 2013.

The role of neurotransmitters and their receptors as immune regulators is an emerging field of research. The first international meeting on nerve-driven immunity with reference to the impact of neurotransmitters and neuropeptides on the immune system, chaired by the pharmacologist Marta Weinstock [Jerusalem, Israel] as a satellite symposium, suggested to coin the novel term of 'Neuroimmuno'Transmitter'. Researchers from various countries presented updates on adrenergic and noradrenergic modulation of immune cells and on the role of the cholinergic system in modulating various aspects of immune function suggesting cholinergic agents as future immunomodulators. Dopamine and dopamine receptors, involved in several autoimmune processes, have potent effects on endogenous immune production, whereas opioid modulation of the innate immune response may be implicated in the susceptibility to infection and wound healing; their suppressing effects on proinflammatory responses may result in suppressed wound healing. GABA, the principal inhibitory neurotransmitter in the brain, is another immunomodulatory molecule with effects on immune cells, in particular $\mathrm{T}$ cells, and a group from the Karolinska Institute (Stockholm, Sweden), reported on GABA-secreting dendritic cells, their GABA receptors being activated by GABA, which results in increased migration as a possible activation function in the immune system, promoted by intracellular pathogens [1].

\section{Financial \& competing interests disclosure}

The author has no relevant affliations or financial involvement with any organization or entity with a financial interest in or financial conflict with the subject matter or materials discussed in the manuscript. This includes employment, consultancies, honoraria, stock ownership or options, expert testimony, grants or patents received or pending, or royalties.

No writing assistance was utilized in the production of this manuscript.

\section{Reference}

1 Levite M (Ed.). Nerve-Driven Immunity: Neurotransmitters and Neuropeptides in the Immune System. Springer, NY, USA (2012). 